\title{
DISSECTING ANEURYSM OF THE AORTA
}

DIFFERENTIAL DIAGNOSIS FROM SYPHILIS*

\author{
BY \\ G. O. HORNE \\ From the Department of Venereal Diseases, General Infirmary, Leeds
}

Up to 1934 only six of the 300 cases of dissecting aneurysm of the aorta described in the literature had been diagnosed ante mortem (Shennan, 1934). Many correctly diagnosed cases have since been reported (with decreasing pride) and the condition is now well enough recognized in general medical practice for cases to be reported only when they present some unusual or particularly instructive feature.

Dissecting aneurysm has been wrongly diagnosed ante mortem as syphilitic aortic disease on many occasions and confusion can also occur post mortem. The importance of this differential diagnosis tends to be overlooked, especially in the standard textbooks on syphilology and cardiology, and even in recent monographs on syphilitic cardiovascular disease. If the current decline in the incidence of syphilis continues, there is likely to be a relative increase in the incidence of dissecting aneurysm-a phenomenon that has already been noted (Simpson, 1947; Newcomb, 1953).

A personal, salutary experience has prompted a review of the subject from this point of view. The pathogenesis, symptomatology, and diagnosis of dissecting aneurysm are briefly described first of all (giving references for further study), three illustrative cases are reported, and the differential diagnosis from syphilis is then discussed.

\section{Definition of Dissecting Aneurysm}

A dissecting aneurysm is the lesion produced by penetration of the circulating blood into the substance of the wall of a vessel with subsequent extension of the effused blood for a varying distance between its layers. It most cases the aorta is the vessel involved, though dissecting aneurysms can form in the pulmonary artery and in all grades of arteries down to the small perforating arteries of the brain. (Shennan, 1934).

Incidence.-The exact incidence of dissecting aneurysm of the aorta is not known, but the literature reveals an incidence of about $0 \cdot 1$ to 1 per cent. in different series of autopsies. The sex incidence shows a preponderance in males of

* Received for publication December 24, 1953. approximately 3 to 1 . The greatest age incidence is between 40 and 60, but Schnitker and Bayer (1944) reviewed 141 cases under the age of 40 , and of the 85 in the series of Gore and Seiwert (1952), 32 were under this age.

Pathogenesis and Mechanism of Dissection.Some controversy still exists about the pathogenesis and mechanism of dissection, and the problem has been fully reviewed by Sailer (1942), Leitch (1944), Gore (1952, 1953), and Gore and Seiwert (1952). It is agreed, however, that pre-existing damage to the medial coat of the aorta is necessary before dissection can occur. This is usually of a degenerative nature, and several different types of "medial necrosis" have been described since the original studies of Erdheim $(1929,1930)$. Hypertension is sometimes associated, but is not an essential factor (for example, it was present in only three out of 32 cases under 40 years of age described by Gore and Seiwert, 1952). Syphilis almost certainly plays no part in the aetiology ; arteriosclerosis may do so. There appears to be a significant coincidence of dissecting aneurysm and coarctation of the aorta and arachnodactyly (see especially Gore, 1953), and pregnancy. Evidence is accumulating that the underlying pathology may be an interference with the vascular supply of the aortic wall ; the possible significance of ischaemia of the media due to changes in the vasa vasorum is discussed later below. Gore (1953) has suggested the possibility of a metabolic basis.

Dissection nearly always begins in the first part of the aorta. There is splitting or separation of the media, usually in its external third. A tear in the intima is not always present, and it is assumed that usually, if not always, bleeding originates in the vasa vasorum (see especially Gore, 1953). The dissection usually spreads distally, occasionally proximally, and its extent varies considerably ; it may also involve any of the branches of the aorta, and extend as far as the popliteal arteries (Gardner, Galbraith, and Hardwick, 1939). When there is a tear in the 
intima, the fact that it is nearly always transverse, and occurs just above the aortic cusps, may be due to the relative fixity of the aorta at this point.

The aneurysm usually ruptures through the adventitia, but " healing" occurs in about 10 per cent. of cases. If the sac is small, organization of the clot, with scarring, may occur, but more usually in surviving cases the aneurysm is found to have re-entered the circulation somewhere distal to the initial rupture. The new channel becomes lined with endothelium, and a "double-barrelled" aorta (and occasionally a complete " tube within a tube") results. Atheromatous changes and calcification may develop in the new lining.

Symptoms and Signs.-Dissection of the aorta can sometimes be either asymptomatic (as recently stressed particularly by MacCuish, 1953), or so obscure in its presentation as to make ante-mortem diagnosis impossible. Usually, however, the clinical features are characteristic, although a wide range of symptom-complexes may occur,* and other diseases may be mimicked. The symptomatology is the same irrespective of age group.

The onset is sudden, sometimes during or following physical exertion, with very severe and overwhelming pain, often described as "tearing" in character. The pain radiates from the precordium or the centre of the thorax to the back, sometimes up into the neck, and sometimes down to the abdomen and into the legs ; it may, however, be predominantly epigastric. It usually reaches its maximum intensity almost immediately and lasts for hours, occasionally for days, and may recur if there is any extension of the dissection.

Dyspnoea is a common symptom and may occur without much pain. Dizziness, syncope, and collapse are not infrequent. Other symptoms depend partly on the extent and site of the dissection, and are attributable to compression of vessels, such as the coronary, carotid, intercostal, and iliac arteries. Symptoms referable to the central nervous system are fairly frequent and may be an important aid to diagnosis.

If the patient survives and is seen soon after the attack he may be very distressed on account of pain, but is frequently collapsed or even comatose. The respiratory rate may be increased and cyanosis is occasionally present. There is often evidence of hypertension, and the blood pressure remains high after the dissection has occurred, or falls during the period of shock and recovers later. A systolic aortic murmur may be present, and in cases surviving more

* These have recently been reviewed in detail by Baer and Goldburgh, (1948); and by Levinson, Edmeades, and Griffith, 1950). than a few hours an aortic diastolic murmur and peripheral evidence of aortic incompetence may develop. The latter is a very important sign, and is discussed in detail below. Retrosternal dullness may be increased and a pulsating mass may be detected at the back of the thorax.

Many other varied physical signs may be present, depending on the extent of the dissection and on which branches of the aorta are compressed. The very close correlation between clinical syndromes and the pathological anatomy has been well demonstrated in the analysis by Warren and McQuown (1948). A wide range of neurological syndromes may occur. These have been reviewed by Weisman and Adams (1944), who described three principal types of syndrome due to ischaemia of the peripheral nerves, of the spinal cord, and of the brain ; and by Moersch and Sayre (1950), who found evidence of neurological involvement in nearly half of a series of 26 cases.

Radiological examination may reveal an enlarged heart, and sometimes widening of the aortic shadow, which may gradually or rapidly increase in size. There may be diminished or absent aortic pulsation. (The $x$-ray findings have been reviewed by Wood, Pendergrass, and Ostrum, 1932 ; and by Levinson and others, 1950). Electrocardiograms may show evidence of hypertension or myocardial disease, and sometimes the changes of coronary infarction. There may be moderate or slight fever and leucocytosis for a few days after the dissection has occurred.

Course of the Disease.-Death may occur immediately, or within a few minutes or a few days, through rupture of the aneurysm into the pericardium, mediastinum, or lungs, or through obstruction of a vital artery. About 20 per cent. of cases survive the initial attack, but some of these die soon after, either after a further dissection or through heart failure. About 10 per cent. survive for longer periods and may live a normal life for many years. ${ }^{*}$ Such surviving cases are usually called " healed" or "chronic". Death may ultimately be due to unrelated disease, but more usually to progressive cardiac failure following aortic incompetence or hypertensive heart disease, or, rarely, to long delayed rupture of the aneurysm. Treatment has little influence on the course of the disease.

\section{Differential Diagnosis}

The attack often simulates coronary thrombosis, and this is the most common error in diagnosis made during the acute stage. The tearing nature of the

* One case is reported as having lived for 30 years after the development of a dissecting aneurysm (Graham, 1886). 
pain and its distribution should be helpful, however, and a persistently elevated blood pressure is unusual in coronary thrombosis. The fever and leucocytosis may be confusing. The absence of characteristic changes in the electrocardiogram is valuable evidence against coronary thrombosis, but changes in dissecting aneurysm are sometimes highly suspicious of coronary thrombosis, and, in occasional cases, characteristic. This may occur particularly when there is proximal dissection of the aneurysm, and the mechanisms involved have been reviewed by Bourne and Mills (1946) and Oram and Holt (1950). The differential diagnosis from coronary thrombosis has been discussed in detail by White, Badger, and Castleman (1934). In view of the almost universal use of anticoagulants in the treatment of coronary thrombosis, and their contraindication in dissecting aneurysm, this differential diagnosis has become of paramount importance-a case misdiagnosed as a pulmonary embolism and treated with dicoumarol has already been described (Evans, 1944).

Pulmonary or peripheral embolism may have to be considered in the differential diagnosis (see especially Fraenkel and Neil, 1950), and many cases have erroneously been called cerebral-vascular accidents. Confusion with acute surgical conditions, especially of the abdomen, may occur (see especially Blain and Gerbasi, 1948). "Healed" or " chronic" cases, especially when the patients are relatively young, have sometimes been misdiagnosed as having rheumatic heart disease (Etter and Glover, 1943 ; Levine, Stein, Gordon, and Mitchell, 1951).

An erroneous diagnosis of syphilitic cardiovascular disease has frequently been made ante mortem in both acute and chronic cases of dissecting aneurysm, and confusion can arise even at autopsy. Even negative serological tests have not always prevented an erroneous diagnosis of syphilis, since it is known that these tests may occasionally be negative in cardiovascular syphilis. In some cases, even when syphilis was accepted as being an unlikely explanation, antisyphilitic treatment has been given.

Confusion with Syphilis.-Three recent case histories from the Department of Venereal Diseases at the General Infirmary at Leeds illustrate the confusion which may arise.

Case 1, a married man aged 31, was referred by a physician in 1942 . The only pertinent history recorded at that time was of increasing breathlessness on exertion and recurrent attacks of dizziness, which had been present for about 18 months. He had had gonorrhoea at the age of 17 , but there was no history of a genital sore. There was no history of rheumatism.
Examination.-The heart was found to be enlarged, there were blowing systolic and diastolic murmurs in the aortic area, and the blood pressure was $130 / 60$. The radial pulse was not collapsing in type. $X$ ray of the chest showed left ventricular enlargement and there was considerable prominence of the ascending aorta suggesting syphilitic aortitis. The blood Wassermann reaction was "strongly positive", and a diagnosis of syphilitic aortic incompetence was made. No other clinical evidence of syphilis was found, except a small healed penile scar.

Further Developments.-Between October, 1942, and June, 1946, the patient received a large amount of antisyphilitic treatment with potassium iodide, neoarsphenamine, and bismuth. The blood Wassermann reaction was "slightly positive" in September, 1944, and June, 1945, and " negative" on several occasions during each of the years 1946 to 1950 . In July, 1946, the cerebrospinal fluid showed no abnormality.

A Corrigan-type pulse was observed to develop in the left arm, and from the beginning of 1947 a marked difference in the blood pressure readings in the two arms was repeatedly recorded : on the left side the systolic pressure varied between 140 and 150 and the diastolic between 40 and 50 , and on the right side the readings were 105 to 110 and 80 respectively. The aortic murmurs became more pronounced.

Dyspnoea on exertion became more easily provoked and attacks of dizziness persisted. An electrocardiogram at the end of 1949 showed slight impairment of conduction from auricle to ventricle but no other abnormality. $X$-ray at this time showed that there had been some increase in the aneurysmal dilatation of the ascending aorta ; no calcification was seen in the wall of the vessel.

At a review in the middle of 1951 it was obvious that cardiac decompensation was advancing. A reconstruction of the clinical history at that time revealed that the original symptoms had been preceded by an acute attack of precordial pain, recorded at the time of the review as "suggestive of coronary thrombosis", but no details of this attack were elicited.

Terminal Heart Failure.-The patient was admitted in March, 1952, with severe congestive heart failure. He was coughing up heavily blood-stained sputum, and the possibility that the aneurysm was leaking was considered. In spite of digitalization and other supportive measures his condition rapidly deteriorated, and he died a week after admission.

Post-mortem Findings.-A healed dissecting aneurysm of the aorta extended from a point $1.5 \mathrm{~cm}$. above the aortic ring as far as the origin of the left subclavian artery, and involved the first $3 \mathrm{~cm}$. of the right common carotid artery. The aortic valve cusps were enlarged but otherwise normal, and the orifice admitted four fingers. There was marked dilatation and hypertrophy of the left ventricle. There was some fresh blood-clot in the trachea and both main bronchi. The aneurysmal sac was in direct contact with the right main bronchus and, though no direct communication could be found, the presence of blood and blood-clot in the bronchus 
indicated a communication. The coronary arteries showed patchy, non-constrictive atheroma, but were otherwise normal. There was no evidence suggestive of healed or active syphilis.

The aortic valve cusps appeared histologically normal. The split in the aorta had occurred between the inner two-thirds and the outer one-third of the medial coat, so that the inner tube was composed of intima and the inner two-thirds of media, the outer tube of the outer third of media and adventitia. In the ascending aorta proximal to the split were small areas of damage in the media in line with the split, but these may only have been artifacts. There was a thick layer of oedematous fibrous tissue around the split, and this extended to form a partial lining to the outer sac. There was no evidence of any inflammatory process, apart from a light lymphocytic infiltration confined to the neighbourhood of the vasa vasorum, and the whole microscopic appearance did not suggest active or healed syphilis. The coronary arteries showed the changes of atheroma, but there was nothing suggestive of active or healed syphilis.

Comment.-This patient was diagnosed and treated as a case of syphilitic aortitis (aortic incompetence and aortic aneurysm) on the strength of clinical signs, radiological appearances, and a positive blood Wassermann reaction. He died 10 years later of heart failure and rupture of a long-standing dissecting aortic aneurysm. No evidence of syphilis was found post mortem, and there is no reason to believe that the patient had ever had syphilitic aortitis. If more careful attention had been paid to the clinical history, a correct diagnosis might have been made initially, although the positive blood Wassermann reaction would have aggravated the problem of differential diagnosis. In addition, the relatively rapid reversal of the Wassermann reaction which occurred in this case is unusual in established cardiovascular syphilis, and is more suggestive of an early latent infection. The original incident, interpreted a year before death as a possible coronary thrombosis, was probably the dissection. No evidence of coronary artery disease was found in the electrocardiogram or at autopsy.

Case 2, a married man aged 52, was perfectly well until one day in April, 1951, when, while working with a cement mixer (as a labourer), he suddenly complained of breathlessness and began to cough. In giving his history he played down his symptoms, but it appears that he virtually collapsed, although he was later able to return home by himself. He denied having experienced any pain at the time of this incident. Within a day or two he became hoarse, and the cough became more persistent and irritating, but remained unproductive. He remained at home under the care of his doctor, and was treated as a case of bronchitis.

About 6 weeks after the incident he began to complain of pain unrelated to exercise in the left side of the chest and left arm (dull and aching in character, but also described as if the arm were "leaving him" or as if it " did not belong to him "). Breathlessness and palpita- tion on exertion developed, and the cough became " whistling". In July, 1951, he was sent to hospital.

Examination.-He was hoarse and there was mild stridor. There was some fullness in the neck, with distension of superficial veins over the upper part of the chest (which also showed evidence of collateral circulation developing) and in the left arm. The left arm and hand were markedly colder than the right, and there was clubbing of the fingers of the left hand. The right carotid pulsation was easily palpable, the left impalpable.

No abnormality in the cardiac size was found, and apart from a blowing systolic aortic murmur, no abnormality was detected in the heart. There was marked inequality of the radial pulses, and the blood pressure readings were right $240 / 140$, left $120 / 100$. There was no peripheral oedema or other evidence of cardiac failure ; air entry into the lungs was good, and there were no adventitious sounds. There were no pupillary abnormalities, and examination of the central nervous and other systems revealed no abnormality. Both vocal cords moved well.

$X$ ray showed enlargement of the heart in its transverse diameter, and considerable enlargement of the aorta upwards and to the right; there was no calcification in the vessel wall ; on screening slight pulsation was seen, and the appearances were in keeping with an aneurysm. Electrocardiogram showed left axis deviation, but no evidence of coronary infarction. The blood Wassermann reaction was strongly positive on two occasions.

Diagnosis.-Syphilitic aortic aneurysm, with compression of the left carotid and subclavian vessels.

Treatment.-Potassium iodide and bismuth was followed at a later date with penicillin, and then with more bismuth.

Further Developments.-Exercise tolerance gradually decreased, pain in the left arm on exertion persisted, paroxysmal cough and stridor became more distressing, and the feeling of fullness in the neck became worse. At a review in October, 1952, the possibility that the aneurysm was a dissecting and not a syphilitic one was considered, and further investigations undertaken. The blood Wassermann reaction was still strongly positive.

Tomography Report.-The aneurysm shows best at 9 and $11 \mathrm{~cm}$., but is clearly defined at 7 and $13 \mathrm{~cm}$. It appears to sit on top of the arch from which it presumably arises. It is not possible to say how much the left subclavian is affected, but at $7 \mathrm{~cm}$. there is a shadow suggestive of a normal subclavian. The trachea is considerably compressed and displaced to the right just below the aneurysm. The kinking of the ascending aorta is very well demonstrated at $7 \mathrm{~cm}$.

Angiocardiogram.-A saccular dilatation of the arch of the aorta, with prolongation of the swelling at the base of the left subclavian artery. No medium was seen to enter the left vessels.

Operation.-Following discussion with Mr. P. R. Allison it was agreed that decompression of the mediastinum (resection of manubrium sterni) would 
give relief of symptoms. At operation on January 6, 1953, the trachea was found to be displaced by a large aneurysm which bulged up into the root of the neck. The innominate artery was curved round the right side of this anteriorly. The left common carotid and left subclavian arteries could not be felt with certainty.

Results.-The operation was followed by considerable relief of pressure symptoms, but about 9 months later he began to complain again of a feeling of tightness in the neck, throbbing, and pain in the left shoulder. This was followed by sudden onset of hoarseness. He was found to have a left recurrent nerve palsy, and it was decided that no further treatment was indicated.

Comment.-It seems probable that this case was also one of dissecting aneurysm of the aorta in spite of the positive blood Wassermann reaction, but it is unlikely that it will be possible to make a definite diagnosis ante mortem. The sudden onset of symptoms and their nature, especially in the absence of previous symptoms, favour a dissecting aneurysm rather than syphilis. Compression symptoms do occur with syphilitic saccular aneurysms, and the subsequent history of the patient and the results of the investigations carried out are compatible with both conditions.

Case 3, a married man aged 49 , referred by a physician in January, 1953, had begun about a year before to complain of a dull gnawing pain in the left upper chest and shoulder region, which he attributed to working in a draught. This lasted for several months, then cleared up spontaneously. It recurred the next winter, and prompted him to visit his doctor, who treated him initially for fibrositis. This pain was not related to exercise and did not radiate, but was aggravated by coughing. He tended to play down his symptoms, and there was probably some degree of impaired exercise tolerance.

Questioned about his early medical history, he stated that about 5 years previously, whilst putting a pan on the gas stove, he felt faint and collapsed to the floor. He was helped to a couch and when the doctor arrived he was found to have weakness of one side of the face. No other symptom was complained of (and in particular he denied any painful sensation), and he stated that no other abnormality was noted at the time. He was confined to the house for a period (unknown) and recovered completely. There was no history of rheumatism. He had " fits" when he was a baby, and recurrent attacks of inflammation in both eyes which had started in early childhood and persisted until 1944.

Examination.-He appeared healthy. There was extension to the left of the normal area of cardiac dullness in the second left intercostal space; the apex beat was in the 6th intercostal space in the midclavicular line; there were loud blowing systolic and diastolic murmurs in the aortic area. There was no evidence of cardiac failure or of pressure phenomena. The blood pressure was 100/70 in both arms. No difference between the carotid pulses was detected. There were no abnormalities in the central nervous system (no evidence of residual facial weakness was obtained) or elsewhere in the body, except the eyes, both of which showed scars of interstitial keratitis (patent blood vessels were seen on slit lamp examination). No other clinical evidence suggestive of congenital syphilis could be obtained (he was edentulous).

The blood Wassermann reaction was strongly positive, and the cerebrospinal fluid Wassermann reaction negative.

Electrocardiogram.-Showed changes suggestive of slight myocardial damage, but no evidence of coronary thrombosis.

$X$ ray.-Showed normal lung fields, but enlargement of the ascending part of the arch of the aorta, consistent with an aneurysm (Fig. 1, opposite). There was no calcification in the wall of the vessel. Screen examination confirmed the aneurysmal dilatation of the ascending aorta and first part of the arch.

Angiocardiogram.-This was unsuccessful for technical reasons, but revealed that the dilatation was probably fusiform.

His wife was healthy, and had two healthy children, and a satisfactory obstetric history. Her blood Wassermann reaction was negative.

Diagnosis.-Syphilitic aortic aneurysm and aortic incompetence, with the reservation that the condition might be a dissecting aneurysm.

Treatment.-Three weekly injections of bismuth and then 10 million units of penicillin, followed by a further seven injections of bismuth.

Further Developments.-In June, 1953 he admitted that he felt very much better than before starting treatment ; he felt breathless only on going up hill, suggesting that this symptom had previously been more easily precipitated than he had been prepared to admit. There was no alteration in the clinical signs. He continued weekly injections of bismuth, but failed to return after the fourth injection. He was not seen again until September, when it was discovered that he had had a "heart attack" at the beginning of July. He had wakened up at 2 a.m. extremely breathless, with coughing but no pain. The breathlessness passed off in a few hours, and he remained in bed under the care of his own doctor for several weeks. On examination in September he was found to have marked tachycardia, and the apex beat was very diffuse, and well outside the midclavicular line. The electrocardiogram showed no change from the preceding record. $X$-ray showed that the prominence of the ascending aorta had increased and a separate sac was seen overlying the aortic knuckle (Fig. 2, opposite); there was a small pleural reaction at the base of the left lung. The patient declined to have further investigations carried out.

Comment.-There is no doubt that the patient had congenital syphilis - the ophthalmic history and examination were unequivocal. It is doubtful whether clinical aortic disease ever occurs in congenital syphilis, and it is unlikely that he acquired syphilis later in life, even if this is accepted as being possible in a congenital 


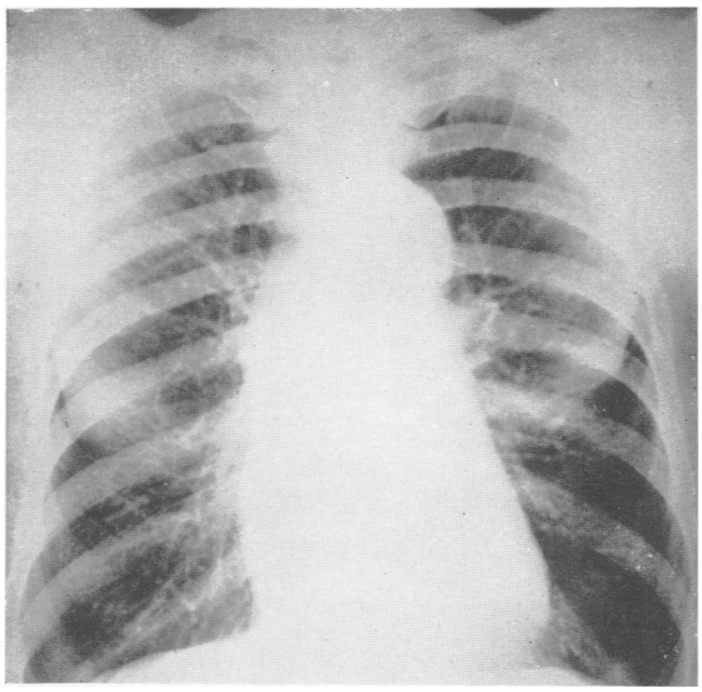

FIG. 1.-Case 3, aged 49, $x$-ray in January, 1953, showing broadening of aortic shadow.

syphilitic. It is difficult to explain the incident 5 years previously in view of the fact that there was complete spontaneous recovery and no recurrence. There was no evidence of generalized vascular disease or neurosyphilis, and he had had no antisyphilitic treatment after the incident. The attack could have been an aortic dissection, and subsequent findings are not incompatible with this. Whilst the more recent acute episode might have been due to an attack of left ventricular failure, it could have been due to a further dissection in the aneurysm, although of course it would be exceptional if he had survived two dissections (the relatively low blood pressure may be of significance here). The serial $x$-ray examinations are more compatible with dissection than with a syphilitic aneurysm.

It seems probable, therefore, that this case too was one of dissecting aneurysm of the aorta in spite of the positive blood Wassermann reaction, but a definite diagnosis is unlikely ante mortem.

Confusion Between Syphilis and Dissecting Aneurysm ante mortem.-This can easily occur in acute and chronic cases of dissecting aneurysm, since in both diseases there may be evidence of aortic incompetence (including the peripheral signs) and aortic aneurysm. The frequency of the occurrence of aortic diastolic murmurs in dissecting ancurysms can be judged by the fully documented reports of Flaxman (1942), David, McPeak, Vivas-Salas, and White (1947) and Levinson and others (1950), in which such murmurs werc present in 21 out of 62 acute cases, and in fifteen out of 31 chronic cases. Schnitker and Bayer (1944), in an analysis of reports of 141 cases under the age of 40 , found a diastolic

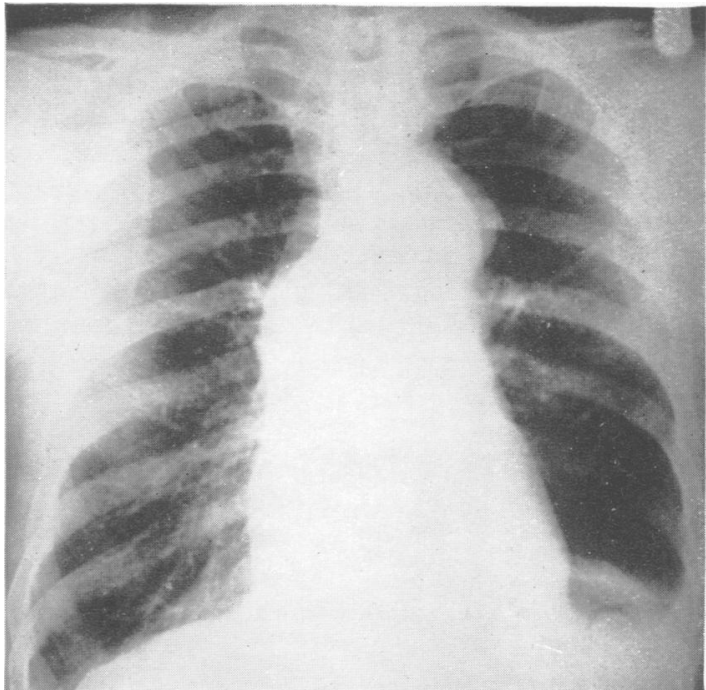

FIG. 2.-Case 3, $x$-ray in September, 1953, showing further broadening of aortic shadow and separate sac overlying aortic knuckle.

murmur interpreted as indicating aortic incompetence in 34 ( 24 per cent.). In most cases peripheral evidence of aortic incompetence was also present.

The discovery of an aortic diastolic murmur just after an incident suggestive of aortic dissection should not nowadays be misleading, especially if the murmur was known not to be present before the incident. Confusion is more likely to arise in cases presenting long after the dissection has occurred, and especially if the latter was relatively silent, or was misinterpreted (as in Case 1 above). The results of serological tests for syphilis must be assessed with discretion. The usually accepted estimate that about 20 per cent. of cases of cardiovascular syphilis have negative serological tests is probably too large, especially in view of the increased sensitivity of tests. In any case, the occurrence of completely negative serological tests in the presence of untreated syphilitic aortic incompetence is probably rare.

In acute cases, care must be taken in the interpretation of praecordial pain. Ischaemic pain occurs in syphilitic aortic disease and might be misinterpreted as being due to dissecting aneurysm ; conversely, the pain of the latter (which may be prolonged) may be misinterpreted as due to syphilis.

Syphilitic aortitis can, of course, be present coincidentally with dissecting aneurysm. For example, of nineteen cases of the latter condition reported by Flaxman (1942), syphilitic cardiovascular disease was diagnosed ante mortem in eight ; three of the eight had positive serological tests for syphilis, and autopsy confirmed that they had syphilitic aortitis. 
Weiss (1938) reported a case in which there seemed to be no doubt that a syphilitic saccular aneurysm and a dissecting aneurysm were both present. In one of the cases described by Gore and Seiwert (1952), cardiovascular syphilis ("substantiated clinically") was present, but was independent of the aneurysm. On the other hand, there may be positive serological tests for syphilis and yet no evidence of syphilitic involvement of the heart or aorta, as in cases of dissecting aneurysm reported by Lundberg (1930) and Boyd and Werblow (1937); one of the two cases of Blain and Gerbasi (1948); two cases of Levinson and others (1950) ; and Case 1 above. Syphilitic aortitis can be present without involvement of the valve, as in two cases of Levinson and others (1950).

Possible Explanations of Aortic Incompetence.Many authors have discussed the possible explanation of the aortic diastolic murmur and aortic incompetence which so frequently develop after dissecting aneurysm and cause confusion with syphilis. Gross enough faults in the aortic valve have usually been vainly sought.

David and others (1947) reported minimal evidence of disease of the aortic leaflets in eight of their sixteen cases (six sclerotic and two inflammatory), but there was no correlation between this and auscultation, since in six cases with a diastolic murmur the leaflets were normal. Gouley and Anderson (1940) reported lipping or thickening of the central portions of the free margins of the valve leaflets, but considered this change secondary to the leak. Levinson and others (1950) found minimal pathological changes in the cusps of three of their sixteen cases. Sando and Helm (1952) described the valve cusps in their case (with dilated ring) as being "fenestrated". Lundberg (1930) attributed the insufficiency of the aortic valve in one case to rupture of the common point of attachment of two cusps to the wall of the aorta. Displacement of cusps as a result of the dissection was reported by Gallavardin and Gravier (1922), Gravier (1924, quoted by Lundberg, 1930), and by Levinson and others (1950) in one case. Spicknall and Binford (1948) noted rupture above a commissure, leading to lowering of that section of the aortic cusp, thus making an incomplete closure although the aortic ring was of normal size. However, Gouley and Anderson (1940) were unable to detect any definite alteration in alignment of the leaflets in any of their classical cases.

Resnik and Keefer (1925) amplified the suggestion originally made by Letulle (1905) that there might be a situation in the circulation analogous to an arterio-venous fistula (known to give rise to peri- pheral signs of aortic incompetence even when the valves are normal) produced by the backflow of blood into the aneurysmal sac during diastole. This has been discredited on several counts : in some cases (as that reported by Hamman and Apperly, 1933) the sac was obviously too small to have this effect ; in other cases (such as one reported by Hamburger and Ferris, 1938) no evidence of connection between the lumen of the aorta and the aneurysm could be found.

It is now generally agreed that the incompetence of the valve is a "relative" one, due to deformity of the ring and interference with its normal functioning as a result of the proximal dissection of the aneurysm, although the exact mechanism is not clear. Nearly all the dissections spread as far as the valve ring (e.g., ten of the sixteen cases of Levinson and others, 1950) or very near to it (e.g., four of the remaining cases of Levinson and others, 1950).

Most authors have reported a perfectly normal looking valve ring, but Hall (1926) stated that in his case it was "somewhat stretched" (circumference $11 \mathrm{~cm}$.) ; Roberts (1939) that it was " moderately dilated" (9 cm.); Gouley and Anderson (1940) that it was dilated in all their six cases, and markedly so in three ; Schnitker and Bayer (1944) that it was "somewhat dilated" ; and Sando and Helm (1952) that the circumference was $13 \mathrm{~cm}$. Levinson and others (1950) considered that the valve ring was sufficiently stretched to account for incompetence in only two of their sixteen cases.

Bizarre aortic murmurs have occasionally been noticed. Roberts (1939) reported a case in which there was a systolic thrill of a peculiar vibrating nature and a long coarse whistling systolic murmur over the aortic area and neck vessels, as well as the usual aortic diastolic murmur. At autopsy he found a prominent shelf-like projection in the aorta just above the valve; the aortic ring was also moderately dilated (see above). Levinson and others (1950) described diastolic murmurs of a musical quality in two of their cases.

Confusion when Aneurysm is Demonstrable.When there is clinical and/or radiological evidence of aneurysmal dilatation of the aorta in dissecting aneurysm, confusion with syphilis may obviously occur, especially if aortic incompetence is also present. Differentiation from a saccular aneurysm may, of course, be impossible, but the rate of development may be informative, and extension of the aneurysm into the branches of the aorta may be diagnostic. Calcification in both the true and false sacs has been demonstrated radiologically by Weiss, Kinney, and Maher (1940), and angiocardiography 
may be helpful (Robb, 1946 ; Golden and Weens, 1949 ; Reich, 1949 ; Dotter and Steinberg, 1951).

Confusion post mortem.-Confusion with syphilis may also occur at post-mortem examination. A dissecting aneurysm may be saccular or fusiform, and in some cases there may be a true aneurysmal dilatation in addition to the intramural dissection (see also below). Several authors have commented on the similarity to syphilis of the naked-eye appearance of the aorta in some cases of dissecting aneurysm, but in none of these could microscopic evidence of syphilis be found. Erdheim (1929) admitted to having used material to illustrate the lesions of syphilis which he later found microscopically to be non-syphilitic.

Graham (1886), Erdheim (1929, 1930), Niehaus and Wright (1941), and Logue (1943) described longitudinal grooves or scars on the intimal surface, sometimes giving a corrugated appearance, which corresponded to medial defects. Rottino (1939) reported a case in which the ascending aorta showed the classical appearance of syphilis-wrinkling and puckering of the intimal surface-but microscopically there was only the degenerative medial change typically associated with dissecting aneurysm. In one of five cases reported by Klotz and Simpson (1932), there was longitudinal wrinkling suggestive of syphilis, but they commented on the absence of characteristic stellate scars, and the depressed areas were found to lie above areas of medial necrosis. In another of their cases, macroscopic changes suggesting syphilis were found, but lacked the typical stellate character. Beresford (1951) stated that in one of his eleven cases the arch of the aorta showed naked-eye changes very suggestive of syphilis, but histological examination disproved this.

Gouley and Anderson (1940) observed the gross appearance of syphilis in three of their six cases. These changes (fine nodularity and scarring) were confined entirely in two cases, and nearly entirely in the third, to the new channel, and they attributed the appearance to the endotheliolization processthe new "intimal" layer being much thicker than that of the usual aorta. Cassidy and Pinniger (1946) observed longitudinal wrinkling of the surface of the false channel in their case. Leitch (1944) stated that confusion may also occur in cases in which the new lumen has become filled with thrombus and undergone scarring : the intima may show the wrinkled and scarred "tree-bark" appearance of syphilis.

An example of possible confusion of this type is seen in Case 10 of Warren and McQuown (1948) ; here there was " an aorta which showed evidence of both syphilis and atherosclerosis. The ascending portion of the arch showed longitudinal wrinkling and an occasional pearly-grey, waxy plaque, as well as a few yellow raised plaques, without ulceration ". The clinical data provided no evidence of syphilis; no report of serological tests or microscopic examination is quoted.

Confusion the other way round may occur occasionally-some cases of ruptured saccular aneurysm due to syphilis may have been wrongly called dissecting aneurysms. For example, in Case 4 of Reich (1944), there was a history of chancre 13 years previously but the blood Wassermann reaction was negative; the aorta revealed the "wrinkling of luetic aortitis ", there was a " tumour-like bulge the size of a baseball just below the diaphragm ", "the outer coat was dissected off for a short distance", and "a perforation about the size of a goose quill was the source of the haemorrhage" (into the peritoneum), " microscopy corroborated the presence of luetic aortitis". There would seem little justification on the evidence presented to call this a dissecting aneurysm, since the adventitia may appear to be "dissected off for a short distance" in a case of ruptured syphilitic saccular aneurysm.

Confusion in the microscopic picture may occur in spite of the conclusion of Sailer (1942) that " common to the great majority of instances [of dissecting aneurysm] is a process which is independent of changes in the vasa vasorum or the intimal coat of the aorta and which is unaccompanied by exudative cellular inflammatory changes. When reacting cells are present, they are usually few and appear to be secondary to degenerative lesions". The possible significance of changes in the vasa vasorum is discussed separately below.

\section{Possible Relationship between Syphilis and Dissecting Aneurysm}

There is general agreement in the literature that syphilis plays little, if any, part in the aetiology of dissecting aneurysm of the aorta. The overall incidence of syphilis in such cases is what might be expected : Shennan (1934), in his analysis of 300 cases, stated that syphilis was "deemed to be present" in twelve, and McGeachy and Paullin (1937) found syphilis in six of a further 127 cases ; Schnikter and Bayer (1944) found syphilis in five of 141 reported cases under the age of 40 ; Warren and McQuown (1948) found that the incidence of syphilis in their series of ten cases approximated to that in the local hospital population.

Some authors have gone so far as to argue that the very nature of the pathological process in syphilis acts as a deterrent to dissection, since the 
fibrosis would prevent splitting of the vessel wall (Shennan, 1934 ; Weiss, 1935 ; Leitch, 1944). Loeschke (1928) drew this conclusion from an analysis of the early German literature, although he himself described a case in which there was a gumma penetrating the wall of the aorta from the adventitia, which he concluded had paved the way for dissection. Weiss (1938) described a case in which dissection had invaded a coincidental syphilitic saccular aneurysm but slightly, whereas it had ripped the surrounding non-syphilitic wall off the aorta; he concluded that the fusion of the layers of the aorta had tended to prevent dissection.

Many cases have been reported in which undoubted syphilitic aortic disease has been present coincidentally with dissecting aneurysm. In some, characteristic medial necrosis was also seen. That syphilis might be incriminated was suggested by Gsell (1928), who claimed that this might be one of the diseases which act as a "toxin" and cause medionecrosis without obvious changes in the vasa vasorum or adventitia. No evidence to support this theory has been forthcoming.

\section{Medial Necrosis in Saccular and Fusiform Aneurysm}

The characteristic cystic medial necrosis described by Erdheim $(1929,1930)$ and others can apparently be associated with non-syphilitic saccular aneurysms. Rottino (1939) reported the case of a woman who died of congestive heart failure at the age of 70 .

The ascending aorta was transformed into a diffusely dilated, non-elastic thin-walled sac $(12.5 \mathrm{~cm}$. in circumference) which ended abruptly in a moderately dilated arch. The descending aorta was likewise moderately dilated.

This is the case referred to above in which the naked-eye appearances were typical of syphilis, but in which the lesion was microscopically similar to that described in cases of spontaneous rupture of the aorta. Beresford (1951) found a small nonsyphilitic saccular aneurysm associated with a large dissecting aneurysm, rupture of the latter being the cause of death. Medionecrosis was seen near both lesions.

Davies (1941) described the case of a man who died of left ventricular failure at the age of 36,10 years after the onset of cardiovascular symptoms. $H e$ had clinical aortic incompetence and $x$-ray evidence of an aneurysm of the ascending aorta. There was no history of rheumatism or syphilis, and the blood Wassermann reaction was negative. At autopsy the ascending aorta was found to be uniformly dilated $(6 \mathrm{~cm}$. in diameter), the wall thinner than normal, and the intima smooth. Histological examination showed cystic medial necrosis.

\section{Significance of Changes in Vasa Vasorum}

Several authors, including Erdheim (1929, 1930), Moritz (1932), and Rottino (1939), noted alterations in the vasa vasorum in cases of medial necrosis of the aorta. Tyson (1931) and Kellogg and Heald (1933) concluded that arteriosclerotic changes in the vasa vasorum were the fundamental causes of medionecrosis. Amromin, Schlichter, and Solway (1948) concluded, from careful microscopic examination of twelve cases, in seven of which they found narrowing of the lumen of the vasa vasorum, that ischaemia of the media was an important factor in the production of the lesion. McCloskey and Chu (1951) also made careful histological studies of seven cases of dissecting aneurysm of the aorta and found changes in the vasa vasorum of all of them. These changes consisted of medial hypertrophy and splitting of the internal elastic membrane of small arteries, medial hypertrophy with intimal proliferation and endothelial swelling of the arterioles, and endothelial swelling and proliferation of the capillaries; of these the arteriolar changes were by far the more prominent. Gore and Seiwert (1952) and Gore (1952) discussed fully the significance of alteration in the vascularity of the media, and, in particular, compared it with the process in syphilis. They consistently noted what they interpreted as an inadequate reparative process alongside the degeneration of the muscular or the elastic laminae of the media. This was " characterized by an increase in the number of vasa, a mild inflammatory infiltration, and the appearance of variable quantities of myxomatous tissue. In contrast to the vascular features of syphilitic aortitis, the vasa are thinwalled and widely patent, and are supported only by considerably weakened media". This mild reaction contrasts with the more severe syphilitic inflammation, with its accompanying endarteritis and fibrous tissue reaction which helps to seal the medial defects. Confusion with syphilis may nevertheless occur, since in dissecting aneurysm the inflammatory character is apparently sometimes more distinct, simulating very closely the histological picture of syphilis (Gore and Seiwert, 1952).

It is therefore not clear yet what role, if any, alterations in the vascularity of the media play in the aetiology of dissecting aneurysm. Medial necrosis in the aorta of animals following obliteration of the vasa vasorum has been produced experimentally (Schlichter, 1946), but this observation is difficult to interpret. If nutritional impairment of this type is an important factor, it is surprising that syphilis, which is characteristically associated with involvement of the vasa vasorum, does not more often lead to medionecrosis. In fact, 
as has been shown, the incidence of syphilis in cases of dissecting aneurysms of the aorta is no greater than that in the general population.

It may be, of course, that the very chronic process of syphilis is less damaging than the extreme narrowing such as might be produced by spasm, even if only short-lived. The arteriolar spasm known to occur in toxaemia of pregnancy and the relatively high incidence of dissecting aneurysm in pregnancy may therefore not be purely coincidental. There is apparently relatively little new vessel formation in dissecting aneurysm, and since the vasa vasorum probably extend no further than the outer layers of the media (Ramsey, 1936), any acute process causing obstruction of these vessels might have a profound effect on the nutrition of the media. A feature of syphilitic aortitis is the formation of new vessels which pass far into the media (Boyd, 1950), a process which might protect the media from the effects of "acute" ischaemia. The site of the commencement of dissection and that of the maximal involvement in syphilitic aortitis correspond with the site where the maximum number of vasa vasorum are seen (Robertson, 1929). It is easy to see a likely connexion between these two observations in syphilis, but the significance in dissecting aneurysm is less obvious.

\section{Summary}

Dissecting aneurysm of the aorta has frequently been mistaken for syphilitic aortic disease. After a description of the principal clinical features of dissecting aneurysm and a review of the beliefs held on its pathogenesis and mechanism, the differential diagnosis from syphilis, both ante mortem and post mortem, is discussed. Three case histories (one with post-mortem report) illustrate the difficulties encountered in differential diagnosis. It is pointed out that if the current decline in the incidence of syphilis continues there is likely to be a relative increase in the incidence of dissecting aneurysm.

I am indebted to Drs J. B. Bittiner and O. Magidson for their contribution to the discussion on the individual cases and on the general problem. The post-mortem examination in Case 1 was carried out by Dr. I. C. P. Dawson.

\section{REFERENCES}

Amromin, G. D., Schlichter, J. G., and Solway, A. J. L. (1948). Arch. Path. (Chicago), 46, 380.

Baer, S., and Goldburgh, H. L. (1948). Amer. Heart J., 35, 198. Beresford, O. D. (1951). Brit. med. J., 2, 396.

Blain, A., and Gerbasi, F. S. (1948). Amer. Heart J., 35, 868.

Bourne, G., and Mills, P. J. W. (1946). Brit. Heart J., 8, 181.

Boyd, W. (1950). " The Pathology of Internal Diseases", 5th ed., p. 91 . Kimpton, London.

Boyd, L. J., and Werblow, S. C. (1937). Ann. intern. Med., 11, 845. Cassidy, M., and Pinniger, J. (1946). Brit. Heart J., 8, 130.

David, P., McPeak, E. M., Vivas-Salas, E., and White, P. D. (1947). Ann. intern. Med., 27, 405.
Davies, D. H. (1941). Brit. Heart J., 3, 166.

Dotter, C. T., and Steinberg, I. (1951). "Angiocardiography" p. 154. Cassell, London.

Erdheim, J. (1929). Virchows Arch. path. Anat., 273, 454.

-(1930). Ibid., 276, 187.

Etter, L. E., and Glover, L. P. (1943). J. Amer. med. Ass., 123, 88.

Evans, J. A. (1944). New Engl. J. Med., 230, 131.

Flaxman, N. (1942). Amer. Heart J., 24, 654.

Fraenkel, G. J., and Neil, J. F. (1950). Lancet, 1, 801.

Gallavardin, L., and Gravier, L. (1922). Paris med., 45, 23.

Gardner, E., Galbraith, A. J., and Hardwick, S. W. (1939). Lancet, 2, 1019.

Golden, A., and Weens, H. S. (1949). Amer. Heart J., 37, 114.

Gore, I. (1952). Arch. Path. (Chicago), 53, 142.

- (1953). Ibid., 55, 1.

- - and Seiwert, V. J. (1952). Ibid., 53, 121.

Gouley, B. A., and Anderson, E. (1940). Ann. intern. Med., 14, 978.

Graham, J. E. (1886). Amer. J. med. Sci., 91, 155.

Gravier, L. (1924). J. Méd. Lyon, 5, 563. (Quoted by Lundberg, 1930).

Gsell, O. (1928). Virchows Arch. path. Anat., 270, 1.

Hall, E. M. (1926). Arch. Path. (Chicago), 2, 41.

Hamburger, M., and Ferris, E. B. (1938). Amer. Heart J., 16, 1.

Hamman, L., and Apperly, F. L. (1933). Int. Clin. (Series 43). 4, 251.

Kellogg, F., and Heald, A. H. (1933). J. Amer. med. Ass., 100, 1157.

Klotz, O., and Simpson, W. (1932). Amer. J. med. Sci., 184, 455.

Leitch, W. H. (1944). Bull. Sch. Med. Maryland, 29, 7.

Letulle. M. (1905). Bull. Soc. méd. Hôp. Paris, 22, 1045.

Levine, E., Stein, M., Gordon, G., and Mitchell, N. (1951). New Engl. J. Med., 244, 902.

Levinson, D. C., Edmeades, D. T., and Griffith, G. C. (1950). Circulation, 1, 360.

Loeschke, A. (1928). Frankfurt. Z. Path. 36, 56.

Logue, R. B. (1943). Amer. J. med. Sci., 206, 54.

Lundberg, A. (1930). Acta med. scand., 73, 19.

MacCuish, R. K. (1953). Brit. med. J., 1, 71.

McCloskey, J. F., and Chu, P. T. (1951). Arch. Path. (Chicago), 52, 132.

McGeachy, T. E., and Paullin, J. E. (1937). J. Amer. med. Ass., 108, 1690.

Moersch, F. P., and Sayre, G. P. (1950). Ibid., 144, 1141.

Moritz, A. R. (1932). Amer. J. Path., 8, 717.

Newcomb, W. D. (1953). British Journal of Venereal Diseases, 29, 67.

Niehaus, F. W., and Wright, W. D. (1941). J. Lab. clin. Med., 26, 1248.

Oram, S., and Holt, M. C. (1950). Brit. Heart J., 12, 10.

Ramsey, E. M., Gaiser, D. W., Carden, G. A., LeCompte, P. M., and Tennant, R. (1936). Yale J. Biol. Med., 9, 14.

Reich, N. E. (1944). Clinics, 3, 346.

(1949). "Diseases of the Aorta. Diagnosis and Treatment", p. 130. Macmillan, New York.

Resnik, W. H., and Keefer, C. S. (1925). J. Amer. med. Ass., 85, 422. Robb, G. P. (1946). "Atlas of Abnormal Angiocardiography." Washington D.C. Army Institute of Pathology. (Cited by Dotter and Steinberg, 1951.)

Roberts, J. T. (1939). Amer. Heart J., 18, 188.

Robertson, H. F. (1929). Arch. Path. (Chicago), 8, 881.

Rottino, A. (1939). Ibid., 27, 320.

Sailer, S. (1942). Ibid., 33, 704.

Sando, D. E., and Helm, S. (1952). Ann. intern. Med., 37, 793.

Schlichter, J. G. (1946). Arch. Path (Chicago), 42, 182.

Schnitker, M. A., and Bayer, C. A. (1944). Ann. intern. Med., 20, 486.

Shennan, T. (1934). Ser. Spec. Rep. med. Res. Coun. (Lond.), No. 193.

Simpson, K. (1947). Lancet, 2, 745.

Spicknall, C. G., and Binford, C. H. (1948). Milit. Surg., 102, 47.

Tyson, M. D. (1931). Amer. J. Path., 7, 581.

Warren, A. S., and McQuown, A. L. (1948). Amer. J. med. Sci., 215, 209.

Weisman, A. D., and Adams, R. D. (1944). Brain, 67, 69.

Weiss, S. (1935). Med. Clin. N. Amer., 18, 1117.

-(1938). New Engl. J. Med., 218, 512.

- Kinney, T. D., and Maher, M. M. (1940). Amer. J. med. Sci., $200,192$.

White, P. D., Badge-, T. L., and Castleman, B. (1934). J. Amer. med. Ass., 103, 1135.

Wood, F. C., Pendergrass, E. P., and Ostrum, H. W. (1932). Amer. J. Roentgenol., 28, 437. 